\title{
Optimizing the CMOS Sensor-Mode for Extreme Linear Dynamic Range MEMS-based CAOS Smart Camera Imaging
}

\author{
Nabeel A. Riza ${ }^{1, *}$, Nazim Ashraf ${ }^{1}$ and Mohsin Mazhar ${ }^{1}$ \\ ${ }^{1}$ School of Engineering, University College Cork, Cork, Ireland
}

\begin{abstract}
Experimentally highlighted are the limitations \& optimizations of the CMOS sensor-mode engaged for the CAOS smart camera. Demonstrated is an optimized method for finding the Camera Response Function (CRF) using a calibrated target.
\end{abstract}

\section{Introduction}

Recently introduced and demonstrated is the CAOS smart camera that not only operates over a very wide optical band (e.g., $350 \mathrm{~nm}$ to $2700 \mathrm{~nm}$ ), it most importantly provides an extreme linear Dynamic Range (DR) reaching $177 \mathrm{~dB}$ [1]. This Pixels of Interest (POI) CAOS smart camera operates by first engaging a multi-pixel sensor (e.g., CMOS/CCD/FPA sensor) for initial lower limited linear DR scene capture that in-turn provides intelligence for the CAOS-mode POI scene capture to produce a net improved extreme linear DR image via image fusion methods.

Linear High Dynamic Range (HDR) imaging is critical for deciphering low contrast targets within HDR scenes, including enhancing multispectral (e.g., color) imaging. Furthermore, accurate and reliable image capture is important for mission critical applications where incorrect image data can lead to catastrophic system failure. The CAOS smart camera inherently meets these stringent requirements and forms a robust machine vision system that can impact several scientific, industrial, and consumer applications. Given many visible light applications, the lower cost CMOS HDR sensor is chosen to design and test the CAOS smart camera. Initial experiments using the $87 \mathrm{~dB}$ HDR sCMOS Quantalux sensor camera model Si 2100-M from Thorlabs has shown the limitations of CMOS sensor cameras versus the CAOS camera when subjected to imaging tests using calibrated HDR test targets with low contrast stepped zones [2]. Current CMOS sensors with specified HDR performance inherently produce a non-linear CRF over the HDR, in particular in the low light and bright light regions. Experiments indeed show such a non-linear CRF prevents a CMOS camera from registering differential output signals with adequate SNR for capture of low contrast targets within a HDR [2]. A classic approach used to enhance digital sensor camera DR is to use multiple uncalibrated synthesized or real images of different exposure times engaged with an algorithm to first generate the CRF and then deploy this full range CRF with real capture multi-exposure images to generate an HDR image that was otherwise not possible using a single exposure limited DR image. It has recently been shown in Ref.3 that by using known calibrated HDR targets to experimentally determine the best estimate of the true CRF, some leading prior-art multi-exposure algorithms produce non-robust HDR images [3]. Part of this nonrobustness comes from the inaccuracy of the deployed $\mathrm{CRF}$ as well the fact that these algorithms engage the full CRF range data that fails to maintain linearity required for ideal multi-exposure HDR image generation operations. Ref.3 also introduced a calibration empowered HDR image generation method that restricts data processing operations to an optimal smaller DR linear CRF range and engages unweighted multi-exposure processing to produce improved robustness image data. This paper highlights further limitations of the deployed CMOS sensor technology and demonstrates additional optimizations for experimental CRF generation, leading to improved accuracy and reliability of the HDR image when compared to HDR image generation using prior-art algorithms.

\section{CMOS Camera CRF Generation and Multi-Exposure HDR Imaging Experiments}

Under bright light $60 \mathrm{KLux}$ illumination conditions using the Image Engineering (Germany) LG3 light box illuminating a 16 patch HDR calibration target with ND filter optimized attenuations, the Thorlabs 16-bit $\mathrm{v}_{\mathrm{p}}$ output signal CMOS camera (using a C-mount GMZ18108 lens) for the brightest (or $0 \mathrm{~dB}$ ) patch starts to trigger pixel saturations (i.e., $\mathrm{v}_{\mathrm{p}}=2^{16}-1=65535$ ) for $0.93 \%$ of pixels in the patch zone for the used exposure time while giving a computed mean $v_{p}$ called $v_{p}(a v g)$ of 39218 . Ideally, this $\mathrm{v}_{\mathrm{p}}(\mathrm{avg})$ should be 65535 as the CMOS sensor received a uniform patch illumination, so all pixels in patch should have produced a $v_{p}=65535$. To counter this experimental anomaly, the raw acquired CRF $\mathrm{v}_{\mathrm{p}}$ data in Ref.3 was

\footnotetext{
Corresponding author: $\underline{\text { n.riza } @ \text { ucc.ie }}$
} 
scaled by a factor of 1.67 , as the $\mathrm{v}_{\mathrm{p}}(\mathrm{avg})=39218$ at the hint of pixel saturation is expected to be at the saturation value of 65535. In reality, such scaling ignores the highly nonlinear behaviour of the deployed CMOS sensor for bright light conditions where the onset of saturated pixel signal outputs has started. Because of this saturated pixel triggering behaviour observed for the deployed CMOS sensor that maybe common place for CMOS \& other digital sensor technologies, this paper proposes an improved CRF generation technique based on pixel $v_{p}$ output signal histogram analysis and calibration target knowledge. Specifically, demonstrated is CRF generation using a $90 \mathrm{~dB}$ 16-patch HDR target similar to ref.3 target design with patch attenuation values in $\mathrm{dB}$ of $0,8,14,18$, $28,32,36,40,44,52,58,64,70,78,84,90$. The camera exposure time is set to $3.703 \mathrm{~ms}$ as it gives the $0 \mathrm{~dB}$ brightest patch $\mathrm{v}_{\mathrm{p}}(\mathrm{avg})$ of 64537 which is very near the 16-bit $\mathrm{v}_{\mathrm{p}}$ limit. Next pixel $\mathrm{v}_{\mathrm{p}}$ value histogram analysis for 16 patches is done that shows that the $28 \mathrm{~dB}$ patch is the first patch of the brighter patches to show no saturated pixels, i.e., no $v_{p}$ values of 65535 .

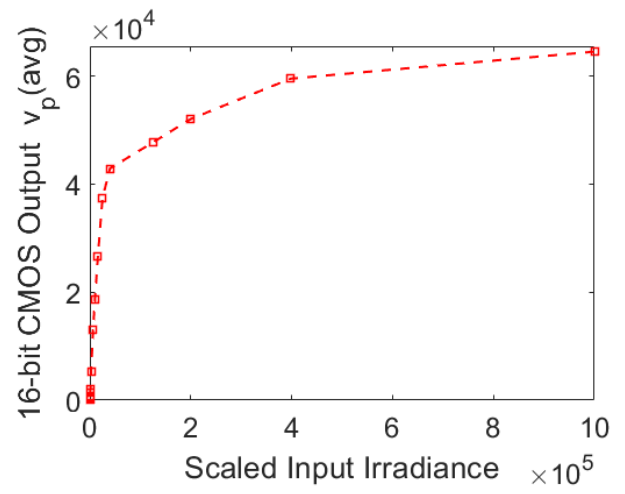

Fig. 1. Experimental CRF.

Let us assume that the scaled input irradiance value $\mathrm{I}_{\mathrm{s}}$ for the zero attenuation or $0 \mathrm{~dB}$ patch is $\mathrm{I}_{\mathrm{s}}=10^{6}$ for the Fig. 1 CRF plot construction. Note that any sufficiently large arbitrary $I_{s}$ should be used to allow accurate slope computations between adjacent data points in plot. The no light patch black region, i.e., for scaled $\mathrm{I}_{\mathrm{s}}=0$ measures a $\mathrm{v}_{\mathrm{p}}(\mathrm{avg})$ of 191. Slope analysis for all data points shows that near continuous linear CRF behaviour is between the $32 \mathrm{~dB}$ patch with a $\mathrm{v}_{\mathrm{p}}(\mathrm{avg})=37486$ and $84 \mathrm{~dB}$ patch with a $v_{p}($ avg $)=389$. Note that for any $v_{p}>64537$, an $I_{s}=10^{6}$ is assigned. Similarly, for any $\mathrm{v}_{\mathrm{p}}<191$, an $\mathrm{I}_{\mathrm{s}}=0$ is assigned. Thus per our ref.3 method, the experimental camera linear dynamic range or $\operatorname{LDR}_{\mathrm{E}}=20 \log (37486 / 389)=39.66 \mathrm{~dB}$ implying that for a near $80 \mathrm{~dB}$ HDR target recovery, only 2 multi-exposure images are needed with a $100 \mathrm{X}$ factor between exposure times. To test the Experimental (E) $\mathrm{CRF}$ and the ref. 3 method, a $78 \mathrm{~dB} 16$ patch HDR target is observed using specific exposure times of $59 \mu$ s and 5.9 $\mathrm{ms}$ that are picked based on the $\mathrm{v}_{\mathrm{p}}(\mathrm{avg}) \mathrm{max} / \mathrm{min}$ selection method in ref.3. Table $1 \mathrm{E} 1$ row shows the measured patch DR values. Unlike our ref.3 method, prior-art methods do not specify the number and increasing time factors for the multi-exposure images, so picked are 4 images starting with the $59 \mu$ s shortest exposure image and 3 other images with 1.5, 15, and 100 increase in exposure time. The Fig.1 CRF is used for all the ref.3 stated 4 prior-art methods, although these methods propose to use un-calibrated target CRFs which would further enhance uncertainty of the HDR image. Table 1 E2 row shows data generated using the 4 images with our ref.3 method.

\begin{tabular}{|c|c|c|c|c|c|c|}
\hline Design (dB) & E1 & E2 & MP & DM & MN & RBS \\
\hline 0 & 0 & 0 & 0 & 0 & 0 & 0 \\
\hline 8 & 10.1 & 9.7 & 11.7 & 12.7 & 3.7 & 14.8 \\
\hline 14 & 15.7 & 15.7 & 17.5 & 17.5 & 10.9 & 20.5 \\
\hline 20 & 21.9 & 22.1 & 24.9 & 24.2 & 26.4 & 28.9 \\
\hline 26 & 26.5 & 26.1 & 29 & 29 & 32.1 & 31.2 \\
\hline 32 & 35.2 & 34.9 & 37.5 & 35.2 & 37.2 & 35.6 \\
\hline 36 & 40.2 & 39.4 & 42.2 & 38.9 & 41.5 & 39.9 \\
\hline 40 & 41.8 & 42.3 & 45.9 & 43.1 & 46.5 & 44.8 \\
\hline 44 & 43.7 & 45.1 & 49.7 & 46.8 & 50.3 & 48.7 \\
\hline 50 & 53.5 & 53.6 & 61.6 & 57.6 & 60.6 & 58.6 \\
\hline 56 & 56.5 & 56.8 & 65.1 & 61.3 & 64 & 61.5 \\
\hline 60 & 61.1 & 62.5 & 69.9 & 67.3 & 70.2 & 66.2 \\
\hline 64 & 64.1 & 66 & 74.2 & 71.3 & 74.3 & 69.1 \\
\hline 68 & 68 & 68.1 & 80.8 & 75.7 & 77.4 & 73 \\
\hline 74 & 73.4 & 73.5 & 86.5 & 82.8 & 84 & 78.5 \\
\hline 78 & 77.4 & 77.4 & 89.3 & 89.9 & 90.8 & 82.4 \\
\hline
\end{tabular}

Table 1. Part 1: CMOS Camera Imaged E1 \& E2 Data using Proposed Method versus 4 Prior-Arts.

\section{Conclusion}

The bright light CRF region of the tested s-CMOS camera shows a highly non-linear response and non-uniform unreliable individual sensor pixel 16-bit voltage output signals despite uniformity of the illumination. Compared to leading prior-art multi-exposure HDR methods, the proposed calibration image deployed CRF generation technique using pixel outputs histogram analysis along with the ref.3 proposed restricted CRF linear zone selection and extraction multi-exposure method experimentally shows higher robustness to non-linearities in CMOS sensor and multi-exposure image fusion techniques

\section{References}

1. N. A. Riza and M. A. Mazhar, " $177 \mathrm{~dB}$ Linear Dynamic Range Pixels of Interest DSLR CAOS Camera," IEEE Photonics Journal (open access), Volume 11, Number 2, April 2, 2019.

2. N. A. Riza and M. A. Mazhar, "Robust Low Contrast Image Recovery over 90 dB Scene Linear Dynamic Range using CAOS Camera," IS \& T Electronic Imaging Conf. Proc. Paper, Jan.26, 2020

3. N. A. Riza and N. Ashraf, "Calibration Empowered Minimalistic Multi-Exposure Image Processing Technique for Camera Linear Dynamic Range Extension,” IS \& T Elect. Imaging Conf. Proc., 2020 